\title{
EXTENSION PROPERTY AND COMPLEMENTATION OF ISOMETRIC COPIES OF CONTINUOUS FUNCTIONS SPACES
}

\author{
CLAUDIA CORREA AND DANIEL V. TAUSK
}

\begin{abstract}
In this article we prove that every isometric copy of $C(L)$ in $C(K)$ is complemented if $L$ is compact Hausdorff of finite height and $K$ is a compact Hausdorff space satisfying the extension property, i.e., every closed subset of $K$ admits an extension operator. The space $C(L)$ can be replaced by its subspace $C(L \mid F)$ consisting of functions that vanish on a closed subset $F$ of $L$. We also study the class of spaces having the extension property, establishing some closure results for this class and relating it to other classes of compact spaces.
\end{abstract}

\section{IntRoduction}

In this article we prove a result (Theorem 2.4) concerning the complementation of isometric copies of $C(L)$ in $C(K)$, for compact Hausdorff spaces $K$ and $L$ satisfying certain assumptions. Our result concerns, in particular, the complementation of Banach subalgebras of $C(K)$ (Corollary 2.5). Indeed, recall that a Banach subalgebra (with unity) of $C(K)$ is the image $\phi^{*} C(L)$ of the isometric embedding $\phi^{*}: C(L) \ni f \mapsto f \circ \phi \in C(K)$, where $\phi: K \rightarrow L$ is a continuous surjection. The Banach subalgebra $\phi^{*} C(L)$ is complemented in $C(K)$ if and only if $\phi$ admits an averaging operator, i.e., a bounded left inverse $P: C(K) \rightarrow C(L)$ for the composition operator $\phi^{*}$. Finding conditions under which a continuous surjection admits an averaging operator is a classical problem in Banach space theory ([1, 5, 13]).

More precisely, Theorem 2.4 deals with the complementation of isometric copies in $C(K)$ of the subspace $C(L \mid F)$ of $C(L)$ consisting of continuous functions vanishing on a closed subset $F$ of $L$.

We observe that the special case of Corollary 2.5 when $K$ and $L$ are compact lines and $\phi: K \rightarrow L$ is a continuous increasing surjection is a corollary of Lemma 2.7 of [8], though the general case (when $\phi$ is not necessarily increasing) is new. By a compact line we mean a linearly ordered set which is compact in the order topology.

Date: October 15th, 2013.

2010 Mathematics Subject Classification. 46B20,46E15,54G12.

Key words and phrases. Banach spaces of continuous functions; extension and averaging operators; complementation of subspaces.

The first author is sponsored by FAPESP (Process no. 2012/25171-0). 
The assumptions about the spaces $K$ and $L$ in Theorem 2.4 are the following: the space $L$ is assumed to be scattered of finite (Cantor-Bendixson) height and the space $K$ is required to satisfy a condition which we call the extension property. We say that $K$ has the extension property if every closed subset $F$ of $K$ admits an extension operator in $K$, i.e., a bounded operator $E_{F}: C(F) \rightarrow C(K)$ such that $E_{F}(f)$ is an extension of $f$, for all $f \in C(F)$.

We note that if $K$ and $L$ satisfy the assumptions of Theorem 2.4 and $C(K)$ contains an isometric copy of $C(L)$ then the space $C(L)$ is actually isomorphic to $c_{0}(I)$, for some index set $I$ (Corollary 3.10). Thus, our Theorem 2.4 can be seen as a Sobczyk-like theorem. The complementation of isomorphic copies of $c_{0}(I)$ in Banach spaces has been studied in [2. More precisely, in [2, Theorem 1.1] it is shown that every isomorphic copy of $c_{0}(I)$ in a Banach space $X$ is complemented if $|I|<\aleph_{\omega}$ and $X$ is isomorphic to a subspace of a $C(K)$ space, with $K$ a Valdivia compactum. We observe that our Theorem 2.4 does not follow from [2, Theorem 1.1], even when $C(L) \cong c_{0}(I)$ with $|I|<\aleph_{\omega}$. Namely, if $K$ is a separable nonmetrizable compact line (say, the double arrow space) then $K$ has the extension property, yet $X=C(K)$ does not satisfy the assumptions of [2, Theorem 1.1]. The latter statement follows from [4, Theorem 2.1] and from the fact that the space of continuous functions on a Valdivia compactum has the separable complementation property ([14, Lemma, pg. 494]).

This article is organized as follows. Section 2 is devoted to the proof of Theorem 2.4. Section 3 is devoted to the study of the extension property. We start Section 3 by stating results that are immediate consequences of well-known facts. Namely, every metrizable compact space and every compact line have the (regular) extension property (Propositions 3.1 and 3.2). Moreover, the extension property is local (Corollary 3.4). Then we study some closure properties of the class of spaces having the extension property. We leave open the question of whether this class is closed under continuous images, but we prove some results in this direction (Proposition 3.5 and Corollary 3.91$)$. We prove also that the class of spaces having the regular extension property is closed under the operation of taking Alexandroff compactifications of arbitrary topological sums (Proposition [3.12). It follows that all spaces that can be assembled from compact lines and compact metrizable spaces by iterating this operation have the extension property. We conclude the section with examples of specific spaces and of classes of spaces that do not have the extension property. More specifically, we show that if $K$ is a ccc space with uncountable spread or if ( $K$ is infinite and) $C(K)$ has the Grothendieck property then $K$ does not have the extension property.

\section{Complementation of isometric copies of $C(L)$ in $C(K)$}

As usual, $C(K)$ denotes the Banach algebra of real-valued continuous functions on the compact Hausdorff space $K$, endowed with the supremum 
norm, and $\mathbf{1}_{K}$ denotes its unity. If $F$ is a closed subset of $K$, we denote by $\rho_{F}: C(K) \rightarrow C(F)$ the restriction operator $\left.f \mapsto f\right|_{F}$ and by $C(K \mid F)$ its kernel.

Definition 2.1. Given a compact Hausdorff space $K$ and a closed subset $F$ of $K$, by an extension operator for $F$ in $K$ we mean a bounded linear map $E_{F}: C(F) \rightarrow C(K)$ that is a right inverse for $\rho_{F}$. We say that $E_{F}$ is regular if $\left\|E_{F}\right\| \leq 1$ and $E_{F}\left(\mathbf{1}_{F}\right)=\mathbf{1}_{K}$ (equivalently, $E_{F}$ is regular if $E_{F}\left(\mathbf{1}_{F}\right)=\mathbf{1}_{K}$ and $E_{F}$ is a positive operator). We say that $K$ has the extension property (resp., regular extension property) if every nonempty closed subset of $K$ admits an extension operator (resp., a regular extension operator) in $K$.

Recall that a bounded operator admits a bounded right inverse if and only if it is onto and its kernel is complemented. Therefore, a closed subset $F$ of $K$ admits an extension operator in $K$ if and only if $C(K \mid F)$ is complemented in $C(K)$. Obviously, the (regular) extension property is hereditary to closed subspaces.

For the proof of the main result of this section, we need two lemmas.

Lemma 2.2. Let $X$ and $Y$ be Banach spaces, $T: X \rightarrow Y$ be a bounded operator, and $Z$ be a closed subspace of $X$. If $T[Z]$ is closed and complemented in $Y$ and $Z \cap \operatorname{Ker}(T)$ is complemented in $X$ then $Z$ is complemented in $X$.

Proof. Let $P$ be a projection of $X$ onto $Z \cap \operatorname{Ker}(T)$ and $Q$ be a projection of $Y$ onto $T[Z]$. The operator $\left.\left(\operatorname{Id}_{X}-P\right)\right|_{Z}: Z \rightarrow Z$ passes to the quotient through $\left.T\right|_{Z}: Z \rightarrow T[Z]$, i.e., there exists a bounded operator $M: T[Z] \rightarrow Z$ with $\left.M \circ T\right|_{Z}=\left.\left(\operatorname{Id}_{X}-P\right)\right|_{Z}$. It is easily checked that $M \circ Q \circ T+P$ is a projection of $X$ onto $Z$.

Lemma 2.3. Let $K$ be a compact Hausdorff space and $S: c_{0}(I) \rightarrow C(K)$ be an isometric immersion, where $I$ is a set. Let $\left\{\xi_{i}: i \in I\right\} \subset C(K)$ be the image under $S$ of the canonical basis of $c_{0}(I)$ and set $G=\bigcap_{i \in I} \xi_{i}^{-1}(0)$. Then the image of $S$ is complemented in $C(K \mid G)$.

Proof. Since $S$ is an isometric immersion, we have $\left\|\xi_{i}\right\|=1$ and thus, for each $i \in I$, there exists $x_{i} \in K$ with $\left|\xi_{i}\left(x_{i}\right)\right|=1$. The fact that $S$ is an isometric immersion yields also that $\left\|\xi_{i} \pm \xi_{j}\right\|=1$ for $i \neq j$ and therefore $\xi_{j}\left(x_{i}\right)=0$ for $i \neq j$. It follows that the points $x_{i}$ are distinct and that all the limit points of the set $\left\{x_{i}: i \in I\right\}$ are in $G$. This implies that, for $\xi \in C(K \mid G)$, the family $\left(\xi\left(x_{i}\right)\right)_{i \in I}$ is in $c_{0}(I)$. Hence the map $P: C(K \mid G) \rightarrow c_{0}(I)$ defined by $P(\xi)=\left(\xi_{i}\left(x_{i}\right) \xi\left(x_{i}\right)\right)_{i \in I}$ is a bounded left inverse for $S$ and thus the image of $S$ is complemented in $C(K \mid G)$.

Theorem 2.4. Let $K$ be a compact Hausdorff space having the extension property. If $L$ is a compact Hausdorff scattered space of finite height and $F$ is a closed subset of $L$ then every isometric copy of $C(L \mid F)$ in $C(K)$ is complemented. 
Proof. First, we observe that we can restrict ourselves to the case when $F$ has at most one point. Namely, if $F$ has more than one point then $C(L \mid F)$ is isometric to $C\left(L_{1} \mid F_{1}\right)$, where $L_{1}$ is the quotient of $L$ obtained by collapsing $F$ to a single point $p \in L_{1}$ and $F_{1}=\{p\}$. Note that $L_{1}$ is again scattered of finite height.

In what follows, either $F=\emptyset$ or $F=\{p\}$. We will use induction on the height of $L$. Denote by $L^{\prime}$ the Cantor-Bendixson derivative of $L$. The case when $F=\{p\}$ with $p \in L \backslash L^{\prime}$ can be reduced to the case when $F$ is empty since $C(L \mid\{p\})$ is isometric to $C(L \backslash\{p\})$.

Let $S: C(L \mid F) \rightarrow C(K)$ be an isometric immersion and denote the restriction of $\rho_{L^{\prime}}: C(L) \rightarrow C\left(L^{\prime}\right)$ to $C(L \mid F)$ by $R: C(L \mid F) \rightarrow C\left(L^{\prime} \mid F\right)$. Setting $I=L \backslash L^{\prime}$, then $\left.f \mapsto f\right|_{I}$ is an isometry from $C\left(L \mid L^{\prime}\right)$ onto $c_{0}(I)$. For $i \in I$, denote by $f_{i} \in C\left(L \mid L^{\prime}\right)$ the characteristic function of $\{i\}$ and set $\xi_{i}=S\left(f_{i}\right)$.

Define $G$ as in the statement of Lemma 2.3. The map $\rho_{G} \circ S$ annihilates the set $\left\{f_{i}: i \in I\right\}$ and thus also its closed span $C\left(L \mid L^{\prime}\right)=\operatorname{Ker}(R)$. It follows that $\rho_{G} \circ S$ passes to the quotient through $R$, i.e., there exists a bounded operator $\bar{S}: C\left(L^{\prime} \mid F\right) \rightarrow C(G)$ such that $\bar{S} \circ R=\rho_{G} \circ S$.

We will prove in a moment that $\bar{S}$ is an isometric immersion. Assuming that this is the case, we will conclude the proof by applying Lemma 2.2 with $T=\rho_{G}$ and with $Z$ equals to the image of $S$. Note that $T[Z]$ equals the image of $\bar{S}$, which is (closed and) complemented in $C(G)$, by the induction hypothesis. Moreover, $Z \cap \operatorname{Ker}(T)=S\left[C\left(L \mid L^{\prime}\right)\right]$ is complemented in $C(K \mid G)$ by Lemma 2.3 and $C(K \mid G)$ is complemented in $C(K)$, because $K$ has the extension property.

Now let us prove that $\bar{S}$ is an isometric immersion. Let $g \in C\left(L^{\prime} \mid F\right)$ with $\|g\|=1$ be fixed. Then $\bar{S}(g)=\left.S(f)\right|_{G}$, where $f \in C(L)$ is an arbitrarily chosen extension of $g$. To prove that $\|\bar{S}(g)\|=1$, we will show that $f$ can be chosen such that $\|f\|=1$ and such that $S(f)$ attains its maximum modulus on $G$. Consider first the case when $g$ admits an extension $f \in C(L)$ with $|f|<1$ on $I$. Assume by contradiction that $S(f)$ attains its maximum modulus at a point $x \in K \backslash G$. Then $\xi_{i}(x) \neq 0$, for some $i \in I$. For $\varepsilon>0$ small enough, we have $\left\|f \pm \varepsilon f_{i}\right\|=1$ and

$$
1=\left\|S\left(f \pm \varepsilon f_{i}\right)\right\| \geq\left|S(f)(x) \pm \varepsilon \xi_{i}(x)\right|>1 .
$$

Finally, let $f$ be an extension of $g$ with $\|f\|=1$. We can assume that the set

$$
J=\{i \in I:|f(i)|=1\}
$$

is infinite, otherwise $f$ could be modified so that $|f|<1$ on $I$. For $i \in J$, we have $\left\|f \pm f_{i}\right\|=2$ and thus there exists $x_{i} \in K$ with $\left|S(f)\left(x_{i}\right) \pm \xi_{i}\left(x_{i}\right)\right|=2$. Since $\left|S(f)\left(x_{i}\right)\right| \leq 1$ and $\left|\xi_{i}\left(x_{i}\right)\right| \leq 1$, we must have $\left|S(f)\left(x_{i}\right)\right|=1$ and $\left|\xi_{i}\left(x_{i}\right)\right|=1$. As in the proof of Lemma 2.3, the points $x_{i}, i \in J$, are distinct and the limit points of $\left\{x_{i}: i \in J\right\}$ are in $G$. Taking a limit point $x$ of the infinite set $\left\{x_{i}: i \in J\right\}$, then $x \in G$ and $|S(f)(x)|=1$, concluding the proof. 
Corollary 2.5. Let $K$ and $L$ be compact Hausdorff spaces and $\phi: K \rightarrow L$ be a continuous surjective map. If $K$ has the extension property and $L$ is scattered of finite height then the Banach subalgebra $\phi^{*} C(L)$ of $C(K)$ is complemented (equivalently, $\phi$ admits an averaging operator).

The following result is an immediate consequence of Theorem 2.4 obtained by letting $L=I \cup\{\infty\}$ be the one-point compactification of the discrete space $I$ and by setting $F=\{\infty\}$. Such result has been stated in [12, Theorem 2.1] in the case when $I$ is countable and $K$ is the double arrow space, though the proof that appears in [12] works also in the general case.

Corollary 2.6. If $K$ is a compact Hausdorff space having the extension property then every isometric copy of $c_{0}(I)$ in $C(K)$ is complemented.

Remark 2.7. The class of compact Hausdorff spaces $K$ for which the thesis of Theorem 2.4 holds is clearly closed under continuous images.

\section{THE EXTENSION PROPERTY}

In this section we study the class of compact Hausdorff spaces having the (regular) extension property. We start by collecting some well-known results about the existence of extension operators.

In [13, §6], a compact Hausdorff space $K$ is called a Dugundji space if for every compact Hausdorff space $L$ containing (a homeomorphic copy of) $K$, there exists a regular extension operator for $K$ in $L$. It is well-known that every nonempty compact metrizable space is a Dugundji space ([13. Theorem 6.6]). The following proposition is an immediate consequence of this fact.

Proposition 3.1. Every compact metrizable space has the regular extension property.

In a compact line, a regular extension operator for a nonempty closed subset can be constructed by decomposing its complement into disjoint open intervals and by using Urysohn's Lemma. One thus obtain the following result.

Proposition 3.2. Every compact line has the regular extension property.

Proof. See [9, Lemma 4.2].

A standard argument using partitions of unity shows that the (regular) extension property is local.

Proposition 3.3. Let $K$ be a compact Hausdorff space and $F$ be a nonempty closed subset of $K$. If every point $x \in F$ has a closed neighborhood $V$ in $K$ such that $V \cap F$ admits an extension operator (resp., a regular extension operator) in $V$ then $F$ admits an extension operator (resp., a regular extension operator) in $K$.

Proof. See [13, Lemma 3.6]. 
Corollary 3.4. Let $K$ be a compact Hausdorff space. If every point of $K$ has a closed neighborhood having the (resp., regular) extension property then $K$ has the (resp., regular) extension property.

3.1. Closure properties of the class of spaces having the extension property. We will first establish that, under some additional assumptions, a continuous image of a space having the extension property also has the extension property (Proposition 3.5 and Corollary 3.9). Then, we show that the class of spaces having the regular extension property is closed under the operation of taking Alexandroff compactifications of arbitrary topological sums (Proposition 3.12).

Proposition 3.5. Let $\phi: K \rightarrow L$ be a continuous surjection, where $K$ and $L$ are compact Hausdorff spaces. Assume that $\phi$ admits a regular averaging operator $P: C(K) \rightarrow C(L)$ (i.e., $P$ is an averaging operator with $\|P\| \leq 1$ ). If $K$ has the (resp., regular) extension property then also $L$ has the (resp., regular) extension property.

Proof. Given a nonempty closed subset $F$ of $L$, let $G=\phi^{-1}[F]$ and $E_{G}$ be a (regular) extension operator for $G$ in $K$. Set

$$
E_{F}=P \circ E_{G} \circ\left(\left.\phi\right|_{G}\right)^{*}: C(F) \longrightarrow C(L) .
$$

That $E_{F}$ is indeed an extension operator follows easily using the fact that, for all $x \in L$, there exists a probability measure $\mu_{x}$ on $\phi^{-1}(x)$ such that $P(h)(x)=\int_{\phi^{-1}(x)} h \mathrm{~d} \mu_{x}$, for all $h \in C(K)$ (see [13, Proposition 4.1]).

The following definition and lemma are required for the proof of Proposition [3.8, from which Corollary 3.9 will follow.

Definition 3.6. Given a compact Hausdorff space $K$, a closed subset $F$ of $K$, and a closed subset $G$ of $F$, by an extension operator modulo $G$ for $F$ in $K$ we mean a bounded operator which is a right inverse for the map $\left.\rho_{F}\right|_{C(K \mid G)}: C(K \mid G) \rightarrow C(F \mid G)$.

Lemma 3.7. Let $K$ be a compact Hausdorff space, $F$ be a closed subset of $K$, and $G$ be a closed subset of $F$. If $F$ admits an extension operator modulo $G$ in $K$ and $G$ admits an extension operator in $K$ then $F$ admits an extension operator in $K$.

Proof. Since $F$ admits an extension operator modulo $G$ in $K$, the subspace $C(K \mid F)$ is complemented in $C(K \mid G)$. Moreover, since $G$ admits an extension operator in $K$, the subspace $C(K \mid G)$ is complemented in $C(K)$. Hence $C(K \mid F)$ is complemented in $C(K)$.

Proposition 3.8. Let $K$ be a compact Hausdorff space and denote by $K^{\prime}$ its Cantor-Bendixson derivative. If $K^{\prime}$ has the extension property and $K^{\prime}$ admits an extension operator in $K$ then $K$ has the extension property.

Proof. If $F$ is a closed subset of $K$ then $F \cap K^{\prime}$ admits an extension operator in $K$. Moreover, the map $E: C\left(F \mid F \cap K^{\prime}\right) \rightarrow C\left(K \mid F \cap K^{\prime}\right)$ defined by 
$\left.E(f)\right|_{F}=f,\left.E(f)\right|_{K \backslash F} \equiv 0$ is an extension operator modulo $F \cap K^{\prime}$ for $F$ in $K$. The conclusion follows from Lemma 3.7.

Corollary 3.9. Let $K$ be a compact Hausdorff scattered space of finite height. If $K$ is a continuous image of a compact Hausdorff space having the extension property then $K$ has the extension property and the space $C(K)$ is isomorphic to $c_{0}(I)$, for some index set $I$.

Proof. Note that $C\left(K \mid K^{\prime}\right)$ is isometric to $c_{0}\left(K \backslash K^{\prime}\right)$ and therefore it is complemented in $C(K)$, by Remark 2.7. Thus, $K^{\prime}$ admits an extension operator in $K$ and $C(K) \cong c_{0}\left(K \backslash K^{\prime}\right) \oplus C\left(K^{\prime}\right)$. The conclusion is obtained using induction on the height of $K$.

Corollary 3.10. If $K$ is a compact Hausdorff space having the extension property and $L$ is a compact Hausdorff scattered space of finite height such that $C(K)$ contains an isometric copy of $C(L)$ then $L$ has the extension property and the space $C(L)$ is isomorphic to $c_{0}(I)$, for some index set $I$.

Proof. Simply observe that, by Holsztyński's Theorem [7], if $C(K)$ contains an isometric copy of $C(L)$ then $L$ is a continuous image of a closed subspace of $K$.

As an immediate consequence of Proposition 3.8, we obtain a useful criterion for verifying if a space of finite height has the extension property.

Corollary 3.11. Let $K$ be a compact Hausdorff scattered space of finite height and denote by $K^{(n)}$ its $n$-th Cantor-Bendixson derivative. If for all $n, K^{(n+1)}$ admits an extension operator in $K^{(n)}$ then $K$ has the extension property.

By the Alexandroff compactification of a locally compact Hausdorff space $X$ we mean $X$ itself, if $X$ is compact, or its one-point compactification $X \cup\{\infty\}$, if $X$ is not compact.

Proposition 3.12. The class of compact Hausdorff spaces having the regular extension property is closed under the operation of taking the Alexandroff compactification of arbitrary topological sums.

Proof. Let $\left(K_{i}\right)_{i \in I}$ be a family of nonempty compact Hausdorff spaces having the regular extension property. Denote by $\bigcup_{i \in I} K_{i}$ its topological sum and by $K$ the Alexandroff compactification of the latter. Let $F$ be a nonempty closed subset of $K$ and set $F_{i}=F \cap K_{i}$. When $F_{i} \neq \emptyset$, let $E_{i}$ be a regular extension operator for $F_{i}$ in $K_{i}$. Let us define a regular extension operator $E_{F}$ for $F$ in $K$.

If $F$ is contained in $\bigcup_{i \in I} K_{i}$, choose an arbitrary $p \in F$ and define $E_{F}$ by setting $\left.E_{F}(f)\right|_{K_{i}}=E_{i}\left(\left.f\right|_{F_{i}}\right)$, if $F_{i} \neq \emptyset,\left.E_{F}(f)\right|_{K_{i}} \equiv f(p)$, if $F_{i}=\emptyset$, and (if $I$ is infinite) $E_{F}(f)(\infty)=f(p)$. If $\infty$ belongs to $F$, define $E_{F}$ by setting $\left.E_{F}(f)\right|_{K_{i}}=E_{i}\left(\left.f\right|_{F_{i}}\right)$, if $F_{i} \neq \emptyset,\left.E_{F}(f)\right|_{K_{i}}=f(\infty)$, if $F_{i}=\emptyset$, and $E_{F}(f)(\infty)=f(\infty)$. In the case when $\infty \in F$, the continuity of $E_{F}(f)$ at the point $\infty$ is easily proven using the fact that $E_{i}$ is a positive operator. 
Corollary 3.13. If $K$ is a scattered compact Hausdorff space of height 2 then $K$ has the regular extension property.

Proof. Such a $K$ is a finite topological sum of one-point compactifications of discrete spaces.

3.2. Negative results about the extension property. We now give a few examples of spaces for which the extension property fails (Examples 3.14, 3.17, and 3.18). We also prove results relating the extension property with cardinal invariants of the topological space $K$ (Corollary 3.16) and with the Grothendieck property of the space $C(K)$ (Proposition 3.19).

Example 3.14. The ladder system space [3, pg. 164] is an example of a scattered compact Hausdorff space of height 3 that does not have the extension property. We recall the relevant definitions. Denote by $\omega_{1}$ the first uncountable ordinal and by $L\left(\omega_{1}\right)$ the subset of $\omega_{1}$ consisting of limit ordinals. Set $S\left(\omega_{1}\right)=\omega_{1} \backslash L\left(\omega_{1}\right)$. Recall that a ladder system on $\omega_{1}$ is a family $\mathcal{S}=\left(s_{\alpha}\right)_{\alpha \in L\left(\omega_{1}\right)}$ where, for each $\alpha \in L\left(\omega_{1}\right)$, we have $s_{\alpha}=\left\{s_{\alpha}^{n}: n \in \omega\right\}$ and $\left(s_{\alpha}^{n}\right)_{n \in \omega}$ is a strictly increasing sequence in $S\left(\omega_{1}\right)$ order-converging to $\alpha$. The ladder system topology $\tau_{\mathcal{S}}$ on $\omega_{1}$ is the one for which the elements of $S\left(\omega_{1}\right)$ are isolated and the fundamental neighborhoods of a limit ordinal $\alpha$ are unions of $\{\alpha\}$ with sets cofinite in $s_{\alpha}$. The ladder system space is the one-point compactification $K_{\mathcal{S}}=\omega_{1}^{\mathcal{S}} \cup\{\infty\}$, where $\omega_{1}^{\mathcal{S}}=\left(\omega_{1}, \tau_{\mathcal{S}}\right)$. We claim that the closed subset $F=L\left(\omega_{1}\right) \cup\{\infty\}$ does not admit an extension operator in $K_{\mathcal{S}}$. Assuming by contradiction that an extension operator $E_{F}$ exists, then, for each $\alpha \in L\left(\omega_{1}\right)$, let $\xi_{\alpha} \in C\left(K_{\mathcal{S}}\right)$ denote the image under $E_{F}$ of the characteristic function of $\{\alpha\}$. By the continuity of $\xi_{\alpha}$, there exists $\phi(\alpha) \in s_{\alpha}$ with $\xi_{\alpha}(\phi(\alpha))>\frac{1}{2}$. The map $\phi: L\left(\omega_{1}\right) \rightarrow \omega_{1}$ is regressive and the Pressing Down Lemma ([10, Lemma II.6.15]) yields a stationary subset $S$ of $L\left(\omega_{1}\right)$ on which $\phi$ is constant and equal to some $\beta \in \omega_{1}$. Then, for each positive integer $n$, we pick distinct elements $\alpha_{1}, \ldots, \alpha_{n} \in S$ and we obtain:

$$
\left\|E_{F}\right\| \geq\left\|\xi_{\alpha_{1}}+\cdots+\xi_{\alpha_{n}}\right\| \geq \xi_{\alpha_{1}}(\beta)+\cdots+\xi_{\alpha_{n}}(\beta)>\frac{n}{2} .
$$

Recall that a topological space $X$ is said to satisfy the countable chain condition (briefly: $X$ is $c c c$ ) if every family of disjoint nonempty open subsets of $X$ is countable.

Proposition 3.15. Let $K$ be a compact Hausdorff space and $F$ be a closed subset of $K$. If $K$ is ccc and $F$ admits an extension operator in $K$ then $F$ is ccc.

Proof. Notice that the image of an extension operator $E_{F}: C(F) \rightarrow C(K)$ is an isomorphic copy of $C(F)$ in $C(K)$. The conclusion follows using the fact that a compact Hausdorff space $K$ fails to be ccc if and only if $C(K)$ contains an isomorphic copy of $c_{0}(I)$ for some uncountable set $I$ (see [6, Theorem 14.26]). 
Corollary 3.16. If a compact Hausdorff space $K$ has the extension property and is ccc then $K$ has countable spread, i.e., every discrete subset of $K$ is countable.

Proof. Notice that if $D$ is an uncountable discrete subset of $K$ then $F=\bar{D}$ is not ccc, since the points of $D$ are isolated in $F$.

Example 3.17. For an uncountable cardinal $\kappa$, the space $2^{\kappa}$ does not have the extension property. Namely, $2^{\kappa}$ is ccc ([10, Theorem II.1.9]) and the characteristic functions of the singleton subsets of $\kappa$ form a discrete subset of $2^{\kappa}$ of size $\kappa$. In fact, we can show that $2^{\kappa}$ is not a continuous image of a compact Hausdorff space having the extension property. Namely, since the ladder system space $K_{\mathcal{S}}$ is a Boolean space of weight $\omega_{1}$, it is homeomorphic to a subspace of $2^{\kappa}$. But $K_{\mathcal{S}}$ is not a continuous image of a compact Hausdorff space having the extension property (Corollary 3.9 and Example 3.14).

Example 3.18. Another example of a scattered compact Hausdorff space of height 3 that does not have the extension property is Mrówka's space (the one-point compactification of the space $X$ constructed in [11]). Namely, Mrówka's space is separable (hence ccc) and has uncountable spread. Then Corollary 3.16 applies.

Recall that a Banach space $X$ has the Grothendieck property if every bounded operator from $X$ to a separable Banach space is weakly compact.

Proposition 3.19. Let $K$ be an infinite compact Hausdorff space. If $C(K)$ has the Grothendieck property then $K$ does not have the extension property. Moreover, $K$ is not a continuous image of a compact Hausdorff space having the extension property.

Proof. If $K$ is infinite then $C(K)$ contains an isometric copy of $c_{0}$. If $K$ were a continuous image of a compact Hausdorff space having the extension property then this copy of $c_{0}$ would be complemented in $C(K)$ (Remark 2.7). But every separable complemented subspace of a space with the Grothendieck property is reflexive.

\section{REFERENCES}

[1] S. A. Argyros and A. D. Arvanitakis, A characterization of regular averaging operators and its consequences, Studia Math., 151 (2002), no. 3, 207-226.

[2] S. A. Argyros, J. F. Castillo, A. S. Granero, M. Jiménez, and J. P. Moreno, Complementation and embeddings of $c_{0}(I)$ in Banach spaces, Proc. London. Math. Soc., 85 (2002), no. 3, 742-768.

[3] A. V. Arkhangel'skii, Topological Function Spaces, Math. and its Appls. (Soviet Series) 78, Kluwer Academic Publishers, (Dordrecht, 1992).

[4] C. Correa and D. V. Tausk, Compact lines and the Sobczyk property, preprint, arxiv.org/abs/1310.1950.

[5] S. Z. Ditor, Averaging operators in $C(S)$ and lower semicontinuous sections of continuous maps, Trans. Amer. Math. Soc., 175 (1973), 195-208.

[6] M. Fabian, P. Habala, P. Hájek, V. Montesinos, and V. Zizler, Banach Space Theory: The Basis for Linear and Nonlinear Analysis, CMS Books in Math., Springer, (New York, 2011). 
[7] W. Holsztyński, Continuous mappings induced by isometries of spaces of continuous function, Studia Math., 26 (1966), 133-136.

[8] O. Kalenda and W. Kubiś, Complementation in spaces of continuous functions on compact lines, J. Math. Anal. Appl., 386 (2012), no. 1, 241-257.

[9] W. Kubiś, Linearly ordered compacta and Banach spaces with a projectional resolution of the identity, Topology Appl., 154 (2007), no. 3, 749-757.

[10] K. Kunen, Set Theory: An Introduction to Independence Proofs, Studies in Logic and the Found. of Math. 102, North-Holland Publishing Company, (Amsterdam, 1980).

[11] S. Mrówka, On completely regular spaces, Fund. Math., 41 (1954), 105-106.

[12] W. M. Patterson, Complemented $c_{0}$-subspaces of a non-separable $C(K)$-space, Canad. Math. Bull., 36 (1993), no. 3, 351-357.

[13] A. Pełczyński, Linear extensions, linear averagings, and their applications to linear topological classification of spaces of continuous functions, Diss. Math., 58 (1968).

[14] M. Valdivia, Projective resolution of identity in $C(K)$ spaces, Arch. Math., 54 (1990), $493-498$.

Departamento de Matemática, Universidade de SÃo PAUlo, Brazil

E-mail address: claudiac.mat@gmail.com

Departamento de Matemática, Universidade de SÃo Paulo, Brazil

E-mail address: tausk@ime.usp.br

$U R L$ : http://www.ime.usp.br/ ${ }^{\sim}$ tausk 